NOTICE: this is the author's version of a work that was accepted for publication in Electroanalytical Chemistry. Changes resulting from the publishing process, such as peer review, editing, corrections, structural formatting, and other quality control mechanisms may not be reflected in this document. Changes may have been made to this work since it was submitted for publication. A definitive version was subsequently published in Electroanalytical Chemistry, Vol. 641 (2010) http://dx.doi.org/10.1016/j.jelechem.2010.01.020 


\title{
The performance of differential pulse stripping voltammetry at micro-liquid-liquid interface arrays
}

\author{
Jörg Strutwolf, Micheál D. Scanlon and Damien W. M. Arrigan*, ${ }^{1}$ \\ Tyndall National Institute, Lee Maltings, University College, Cork, Ireland. \\ * Corresponding author; Email: d.arrigan@curtin.edu.au; fax: +61-(0)8-9266-4699; phone \\ $+61-(0) 8-9266-9735$. \\ 1 Current address: Nanochemistry Research Institute, Department of Chemistry, Curtin \\ University of Technology, GPO Box U1987, Perth, WA 6845, Australia.
}

\section{Abstract}

Microporous silicon membranes were recently introduced to create hexagonally-patterned arrays of micro-scale interfaces between two immiscible electrolyte solutions ( $\mu$ ITIES). In this report we present a simulation study of the application of differential pulse stripping voltammetry (DPSV) using these $\mu$ ITIES arrays for ion sensing. Simulations showed that the stripping current for ion detection was enhanced by use of relatively deep pores (i.e. a low pore aspect ratio) and a viscous organic phase. These factors decrease the speed of escape of the preconcentrated ion from the organic side of the ITIES. The stripping current initially increased steeply with preconcentration time but eventually reached a plateau. Experiments performed using a $\mu$ ITIES array with micropores of radius $26 \mu \mathrm{m}$, depth of $100 \mu \mathrm{m}$ and with a gelified organic phase demonstrated the saturation of the stripping peak with increasing preconcentration time for the DPSV detection of tetraethylammonium ion. The reasons for the saturations are discussed in terms of diffusion coefficients and depth of the micropores. 
Keywords: ITIES, ion transfer, pulsed voltammetry, micropore, array, computational electrochemistry.

\section{Introduction}

Electrochemistry at the interface between two immiscible electrolyte solutions (ITIES) [1-4] is of special interest in analytical chemistry because it enables the measurement of electroanalytical signals of charged analyte species in solution without the need for their oxidation or reduction $[1,5-8]$. Ionised substances can therefore be detected in a label-free manner even if their oxidation/reduction is not easily achieved or is masked by the presence of an interfering substance at solid electrodes, e.g. [8, 9].

In general, electroanalytical performance is improved by miniaturisation of the electroactive surface. The introduction of micron-sized solid electrodes [10] led to major progress in electroanalysis. The analogous introduction of micron-scaled ITIES ( $\mu$ ITIES) also brings several advantages $[11,12]$. The smaller size of the interface results in smaller currents and therefore a reduced Ohmic potential drop especially in the resistive organic phase. The $\mu$ ITIES is subject to increased diffusional transport of the analyte compared to their macroscopic counterparts, resulting in an increased current density and in an enhancement of sensitivity and a lowering of detection limits. The strength of the electroanalytical signal can be amplified if several $\mu$ ITIES are operated in parallel, in the form of an array of $\mu$ ITIES [13].

Recently, arrays of $\mu$ ITIES were developed using micropores fabricated within silicon solidstate membranes [14]. Different array designs were fabricated, possessing between 3 and 120 micropores arranged in an hexagonal pattern with between-pore centre-to-centre distances of $100-1000 \mu \mathrm{m}$ and pore radii between 5 and $25 \mu \mathrm{m}$. The silicon membrane thickness was 100 
$\mu \mathrm{m}$. These were used to form arrays of $\mu$ ITIES to investigate simple and facilitated ion transfer $[14,15]$ and for the detection of dopamine [6], oligopeptides [7], and propranolol [5]. The performance of the $\mu$ ITIES array in a hydrodynamic arrangement was also investigated [16].

Cyclic voltammetry $(\mathrm{CV})$ of ion transfer across the $\mu$ ITIES confined within such solid-state micropore arrays was studied in detail by simulations [15]. The simulation model took into account the geometric properties of the membrane as well as possible interactions of the diffusion zones around neighbouring micropores. By comparing simulation with experiments, it was shown that the ITIES was practically co-planar with the membrane surface on the aqueous side, i.e. the pores were filled with the organic phase. In the experiments, the organic phase was gelified to increase the mechanical stability of the interface and to facilitate the experimental set-up. By comparing experimental and simulated voltammograms, the ratio of the diffusion coefficients of the transferring ion in the organic and aqueous phases was determined. The diffusion coefficient in the organo-gel was reduced by a factor of 8.7 compared to that in the aqueous phase.

Applications of stripping voltammetric techniques at ITIES have been investigated by Senda and co-workers [17-21] and by Lee at et al. [22]. Ohkouchi et al. [17] used a micropipette filled with nitrobenzene in contact with an aqueous phase. Acetylcholine was preconcentrated into the nitrobenzene and stripped back into the aqueous phase. Theoretical considerations predicted a dependence of the stripping peak height on the square root of the preconcentration time and this was in agreement with experiments. Katano and Senda developed a thin film organo-gel/water interface electrode which was applied to stripping analysis of $\mathrm{Hg}^{+}$and $\mathrm{Pb}^{2+}$ ions [18] and polyoxyethylene non-ionic surfactants [19]. The fact that the sensitivity of the 
stripping step can be enhanced if the diffusion of the analyte in the organic phase is diminished by gelification of the organic phase was also exploited by Lee et al., who used a micromachined organo-gel membrane for stripping analysis of choline ions [22].

In our recent work, improved sensitivity was achieved by combination of stripping and pulse voltammetric techniques (e.g. differential pulse stripping voltammetry (DPSV)) at the gelified $\mu$ ITIES array formed in solid state membranes. It was found that DPSV improved the detection limits obtained at the $\mu$ ITIES array for oligopeptides [7] and for the beta-blocker drug propranolol [5] in comparison to linear sweep voltammetry, differential pulse voltammetry (DPV) or linear sweep stripping voltammetry.

In this report, we present a detailed examination of the performance characteristics of DPSV at a $\mu$ ITIES array formed at a microporous membrane. The purpose of the work was to develop an understanding of the application of advanced voltammetric methods at arrays of $\mu$ ITIES for the eventual sensitive detection of biological molecules. The simulation results presented clearly indicate the necessary control parameters for the design of new micropore arrays. The influence on the stripping current of the pore depth, of the ratio of the diffusion coefficients of the analyte in the organic and aqueous phases and of the preconcentration time, were examined by computer simulation. The results show that the combination of DPSV with $\mu$ ITIES array formed at the orifice of relatively deep micropores (100 $\mu \mathrm{m}$ depth) in a solidstate membrane serves as an excellent platform for the electroanalytical detection of charged species. The results of the simulated DPSV curves were compared to experiments with tetraethylammonium ions as the analyte. 


\section{Experimental}

\subsection{Reagents}

All reagents were purchased from Sigma-Aldrich Ireland Ltd (unless indicated otherwise) and used without further purification, with the exception of 1,6-dichlorohexane (1,6-DCH) which was purified according to the published procedure[23]. The aqueous phase electrolyte solution of $10 \mathrm{mM} \mathrm{LiCl}$ was prepared in ultrapure water (resistivity: $18 \mathrm{M} \Omega \mathrm{cm}$ ), obtained from an Elgastat Maxima - HPLC purifier (Elga, UK). The model analyte species studied was the tetraethylammonium cation $\left(\mathrm{TEA}^{+}\right)$prepared as the chloride salt $\left(\mathrm{TEA}^{+} \mathrm{Cl}^{-}\right)$in the $10 \mathrm{mM}$ $\mathrm{LiCl}$ aqueous phase. The concentration of the model analyte in all experiments was $150 \mu \mathrm{M}$. The organic phase electrolyte salt was bis-(triphenylphosphoranylidene) ammonium tetrakis(4-chlorophenyl)borate (BTPPATPBCl), which was prepared as described elsewhere[13]. The organic phase was prepared as a gel [7] and was composed of the organic solvent (1,6-DCH), the organic electrolyte, and low molecular weight poly(vinyl chloride) (PVC, from Fluka).

\subsection{Micropore array}

The micropore arrays were fabricated in silicon using a combination of wet and dry silicon etching to thin the wafers and etch pores through the thinned portions, as described previously [14]. The micropore array design used in this study consisted of eight pores arranged in an hexagonal close-packed array geometry. Each individual pore measured $52 \mu \mathrm{m}$ diameter and the pore-to-pore (center-to-center) distance was $491 \mu \mathrm{m}$. The thickness of the silicon membrane, or pore depth, was $100 \mu \mathrm{m}$. Scanning electron microscopic images of the whole array and single pores were presented previously $[7,14,15]$. 


\subsection{Experimental procedure}

All experiments at the $\mu$ ITIES array were performed using a $\mathrm{CHI} 620 \mathrm{~B}$ potentiostat $(\mathrm{CH}$ Instruments, Texas, USA). The cell set-up, utilizing $\mathrm{Ag} \mid \mathrm{AgCl}$ reference electrodes, was described previously $[7,15]$. The waveform parameters for DPSV at the $\mu$ ITIES array, as previously determined [7], were: step height (incremental voltage): $0.004 \mathrm{~V}$, pulse amplitude: $0.05 \mathrm{~V}$, pulse width: $0.1 \mathrm{~s}$, sampling width: $0.05 \mathrm{~s}$, pulse period: $0.2 \mathrm{~s}$. The initial and final potentials of the DPSV scan were $0.85 \mathrm{~V}$ and $0.3 \mathrm{~V}$, resepectively. The preconcentration step was applied for between 3 and $90 \mathrm{~s}$ at a preconcentration potential of $0.85 \mathrm{~V}$. Before each DPSV measurement, a preconditioning step at $0.3 \mathrm{~V}$ was applied for the same time period as the preconcentration time. This ensures that all analyte was extracted from the organogel phase. For each preconcentration time, background DPSVs were also recorded (i.e. in the absence of the analyte ion in the cell) and used for background subtraction from experiments involving $\mathrm{TEA}^{+}$transfer.

\section{Theory}

The simulation of ion transfer across the ITIES confined in micropore arrays was described in detail recently[15]. A summary is given here. Transport is assumed to occur by diffusion only and is described by a diffusion equation in cylindrical coordinates. A sketch of the computational domain including the boundary conditions is presented in Figure 1. The pore is filled with the organic phase and the ITIES is co-planar with the membrane surface on the aqueous side. Initially (at time $t=0 \mathrm{~s}$ ), the concentration of the transferring ion in the aqueous phase is at the bulk value, $c=c^{\mathrm{b}}$, while in the organic phase the concentration is zero. The space is normalised by the radius of the pore, $r_{\mathrm{a}}$, so that $R_{\mathrm{a}}=1$ is the dimensionless pore radius. The pore depth $l$ in physical length units $(\mu \mathrm{m})$ is converted to the dimensionless pore depth $L$ by $L=l / r_{\mathrm{a}}$. The no-flux boundary condition on the right-side of the domain shown in Figure 1 
allows for interaction with the diffusion zones of the six nearest-neighbour micropores of the hexagonal pore arrangement. Following the diffusion zone approach, which is a valid model for $r_{\mathrm{a}}>1 \mu \mathrm{m}$ [24], the right-side boundary line is located at $r_{\mathrm{d}}=3^{1 / 4}(2 \pi)^{1 / 2} r_{\mathrm{c}}$, where $r_{\mathrm{c}}$ is the center-to-center distance between neighbouring pores in the hexagonal unit cell. With $r_{\mathrm{c}}=491$ $\mu \mathrm{m}$ and $r_{\mathrm{a}}=26 \mu \mathrm{m}$, the normalised value for $r_{\mathrm{d}}$ in the simulation is $R_{\mathrm{d}}=9.5$. It was shown previously[15] that, within the time frame of the experiments, in the array configuration employed here the interaction between diffusion zones is minor and the effect of this on the current at the individual pores is small. Comparison of concentration profiles and currents with simulations for larger $r_{\mathrm{c}}$ values, as well as replacing the no-flux boundary condition at $R=R_{\mathrm{d}}$ with a constant (bulk) concentration condition, shows a slight interaction of diffusion zones in some cases with negligible effect on the current.

The kinetics of ion transfer across the ITIES (dashed line in Figure 1) can be described by a Butler-Volmer-type equation [25-28]

$$
\begin{aligned}
\left.\gamma \frac{\partial C_{\mathrm{o}}(R, Z, t)}{\partial Z}\right|_{Z=0}= & K^{0}\left(C_{\mathrm{o}}(R, Z, t) \exp \left(-\alpha\left(\Delta_{\mathrm{o}}^{\mathrm{w}} \varphi-\Delta_{\mathrm{o}}^{\mathrm{w}} \varphi_{\mathrm{i}}^{0^{\prime}}\right) \frac{Z_{\mathrm{i}} F}{R T}\right)\right. \\
& \left.-C_{\mathrm{a}}(R, Z, t) \exp \left((1-\alpha)\left(\Delta_{\mathrm{o}}^{\mathrm{w}} \varphi-\Delta_{\mathrm{o}}^{\mathrm{w}} \varphi_{\mathrm{i}}^{0^{\prime}}\right) \frac{Z_{\mathrm{i}} F}{R T}\right)\right)
\end{aligned}
$$

which, together with the equation for interfacial flux conservation,

$$
\left.\gamma \frac{\partial C_{\mathrm{o}}(R, Z, t)}{\partial Z}\right|_{Z=0}=-\left.\frac{\partial C_{\mathrm{a}}(R, Z, t)}{\partial Z}\right|_{Z=0}
$$


constitutes the boundary condition at the interface. In Equation (1) and (2), $R$ and $Z$ are space variables scaled by the pore radius, $\gamma=D_{\mathrm{o}} / D_{\mathrm{a}}$ is the ratio of diffusion coefficients of the transferring ion in the organic and aqueous phases, $K^{0}=k^{0} r_{\mathrm{a}} / D_{\mathrm{a}}$ is the dimensionless form of the heterogeneous standard rate constant and $\alpha$ is the transfer coefficient. The transfer of $\mathrm{TEA}^{+}$across the ITIES was found to be reversible on the time scale of voltammetric experiments at macro-interfaces [26, 27] and micro-interfaces [29]. Values of $k^{0}=0.5 \mathrm{~cm} \mathrm{~s}^{-1}$ and $\alpha=0.5$ [30] were used in the simulations. Concentrations of the transferring ion in the aqueous and organic phase are normalised by the bulk concentration in the aqueous phase, $C_{\mathrm{a}}$ $=c_{\mathrm{a}} / c^{\mathrm{b}}$ and $C_{\mathrm{o}}=c_{\mathrm{o}} / c^{\mathrm{b}}$. The Galvani potential difference between the aqueous and organic phases is denoted by $\Delta_{\mathrm{o}}^{\mathrm{w}} \varphi$, and the formal Galvani potential difference (or formal ion-transfer potential) is given by $\Delta_{\mathrm{o}}^{\mathrm{w}} \varphi_{\mathrm{i}}^{0^{\prime}}$. Finally, $z_{\mathrm{i}}$ is the charge of the transferring ion $(+1$ in case of $\mathrm{TEA}^{+}$.

The reported currents are normalised by the limiting current, described by the Saito equation [31], multiplied by the number of pores in the membrane $\left(N_{\mathrm{h}}\right): i_{\lim }=4 z_{\mathrm{i}} \mathrm{F} D_{\mathrm{a}} c^{\mathrm{b}} r_{\mathrm{a}} N_{\mathrm{h}}$. The dimensionless current is then given by

$$
G=\left.\frac{\pi}{2} \int_{0}^{1} \frac{\partial C_{\mathrm{a}}(R, Z, t)}{\partial Z}\right|_{Z=0} R \mathrm{~d} R
$$

The gradient in Equation (3) is evaluated along the interface (the dashed line in Figure 1), which is located at $Z=0$. The dimensionless limiting current is then given by $G_{\lim }=1$. 


\subsection{Computational details}

Simulations were performed using the finite element method (FEM) program package COMSOL Multiphysiscs (version 3.5a, COMSOL Ltd., Hertfortshire, UK), equipped with the chemical engineering module. Free mesh parameters were used at locations where high concentration gradients occur. These locations are the points of the orifices on both sides of the pore and the boundary line of the ITIES. Here a maximum size of the triangular elements of 0.0002 and a factor of 1.1 for element expansion were used. This mesh was tested previously and shown to deliver acceptable limits of errors in the calculated currents[15]. The software's built-in direct solver UMFPACK[32] was used to solve the time-dependent system of equations.

The generation of complex excitation waveforms (e.g. potential-time curves) used in timedependent boundary conditions in the simulation of electrochemical systems has been described by Ludwig et al. [33]. For the simulation of DPSV, a FORTRAN 95 program was written for generating the potential-time profile. The DPSV signal was calculated by superimposing a staircase waveform and a pulse waveform [34], as illustrated in Figure 2. The staircase waveform (Figure 2A) had a step height (incremental voltage) of $\Delta_{\mathrm{o}}^{\mathrm{w}} \varphi_{\mathrm{s}}(\mathrm{V})$ and a step width of $\left(\Delta t_{1}+\Delta t_{2}\right)(\mathrm{s})$, while the pulse waveform (Figure $2 \mathrm{~B}$ ) had a pulse amplitude of $\Delta_{\mathrm{o}}^{\mathrm{w}} \varphi_{\mathrm{a}}$, a pulse width of $\Delta t_{2}$ and a period of $\left(\Delta t_{1}+\Delta t_{2}\right)$. The superimposition of both waveforms resulted in the potential-time profile shown on Figure $2 \mathrm{C}$. The generated potential-time values were saved in a table which was then linked to the COMSOL Multiphysics program. During simulations, linear extrapolation between the tabulated potential-time values was performed by the simulation software to allow for time step adaption to minimise computational errors. The input parameters for the generation of the DPSV potential-time profile were chosen in accordance with the experiment: Initial potential, 
$\Delta_{\mathrm{o}}^{\mathrm{w}} \varphi_{\mathrm{i}}=0.85 \mathrm{~V}$, final potential $\Delta_{\mathrm{o}}^{\mathrm{w}} \varphi_{\mathrm{f}}=0.3 \mathrm{~V}$, the incremental voltage $\Delta_{\mathrm{o}}^{\mathrm{w}} \varphi_{\mathrm{s}}=0.004 \mathrm{~V}$, pulse amplitude $\left(\Delta_{\mathrm{o}}^{\mathrm{w}} \varphi_{\mathrm{s}}+\Delta_{\mathrm{o}}^{\mathrm{w}} \varphi_{\mathrm{a}}\right)=0.05 \mathrm{~V}$, pulse period $\left(\Delta t_{1}+\Delta t_{2}\right)=0.2 \mathrm{~s}$ and the pulse width $\Delta t_{2}=$ $0.1 \mathrm{~s}$. For preconcentration times, $t_{\mathrm{c}}$, during which the potential was held at $\Delta_{\mathrm{o}}^{\mathrm{w}} \varphi_{\mathrm{i}}$, times between $3 \mathrm{~s}$ and $90 \mathrm{~s}$ were applied.

The normalised current, Equation (3), was calculated at each preset time interval (0.005 s). Post-processing of the current-time output from COMSOL Multiphysics involved the subtraction of the current values at the end of the intervals $\Delta t_{1}$ and $\Delta t_{2}$ during each pulse period and was performed by an external FORTRAN 95 routine.

\section{Results and Discussions}

In order to justify and verify some of the model assumptions used, such as $\gamma, \Delta_{\mathrm{o}}^{\mathrm{w}} \varphi_{\mathrm{i}}^{0^{\prime}}$ and geometric features of the micropore array, the comparison of an experimental backgroundsubtracted $\mathrm{CV}$ for the transfer of $\mathrm{TEA}^{+}$ions across the aqueous/gelified organic phase boundary to a simulated voltammogram is first presented. The solid line in Figure 3 shows the experimental $\mathrm{CV}$ recorded at a scan rate of $10 \mathrm{mV} \mathrm{s}^{-1}$. The experimental current was normalised to its limiting current of $2.68 \mathrm{nA}$. The open circles are the simulation results, taking into account a formal ion-transfer potential of $\Delta_{\mathrm{o}}^{\mathrm{w}} \varphi_{\mathrm{i}}^{0^{\prime}}=0.58 \mathrm{~V}$. For this specific case, the shape of the CV can be qualitatively explained in terms of spherical and planar diffusion [14] and has been quantitatively analysed by simulation [15], as has been done previously for a similar configuration by Josserand et al. [35]. The limiting current is affected by the position of the ITIES (co-planar with the membrane at the aqueous side, or recessed within the pore), whereas the back peak is sensitive to the value of the diffusion coefficient of the transferring species in the organic phase. The peak current increases with decreasing diffusion 
coefficient of the analyte ion in the organic phase [15]. Using a $\gamma$ value of 0.115 , as evaluated previously [15], and a diffusion coefficient of $D_{\mathrm{a}}=9.80 \times 10^{-6} \mathrm{~cm}^{2} \mathrm{~s}^{-1}$ for $\mathrm{TEA}^{+}$in the aqueous electrolyte phase [36], therefore gives $D_{\mathrm{o}}=1.13 \times 10^{-6} \mathrm{~cm}^{2} \mathrm{~s}^{-1}$ for $\mathrm{TEA}^{+}$in the gelified 1,6DCH phase employed here. The fit between the simulated and experimental CVs also confirms the geometric properties and dimensions of the microporous membrane which were evaluated by scanning electron microscopy $[7,14,15]$.

The CV in Figure 3 also helps to establish the applied potentials to use in the DPSV waveform. For preconcentration, a potential of $0.85 \mathrm{~V}$ is applied to transfer $\mathrm{TEA}^{+}$from the aqueous phase into the organic phase. For the stripping step, the potential is pulsed from 0.85 $\mathrm{V}$ to $0.3 \mathrm{~V}$. Figure $4 \mathrm{~A}$ gives an example for a complete current-time output from a simulation. The preconcentration time was $t_{\mathrm{c}}=30 \mathrm{~s}$, resulting in an initial current transient and a constant limiting current of $G=1$. After $30 \mathrm{~s}$ the DPV potential waveform is applied, initiating the back-transfer of $\mathrm{TEA}^{+}$from the organic to the aqueous phase. The inset in Figure 4A shows a magnified view of the current response to the potential waveform with a period of $\left(\Delta t_{1}+\Delta t_{2}\right)=$ 0.2 and a pulse width of $\Delta t_{2}=0.1 \mathrm{~s}$. Subtraction of the current at the end of $\Delta t_{1}$ and $\Delta t_{2}$ results in the DPV curve shown in Figure 4B.

The influence of the preconcentration time combined with different values of the pore depth and different ratios of the diffusion coefficients on the DPSV signal height, and hence the detection capability of the DPSV technique using the $\mu$ ITIES array, was next investigated. Figure 5 shows the dependence of the DPSV peak current $\left|G_{\mathrm{p}}\right|$ on the preconcentration time for different values of the normalised pore depth, $L$, specifically $1(l=25 \mu \mathrm{m}), 2(50 \mu \mathrm{m}), 4$ $(100 \mu \mathrm{m})$ and $8(200 \mu \mathrm{m})$ (Figure 5A to $5 \mathbf{D})$. The numbers in brackets are the pore depths $l$, where $l=L r_{\mathrm{a}}$ and $r_{\mathrm{a}}=25 \mu \mathrm{m}$. For each pore depth, DPSV simulations for $\gamma$ values of 0.115 , 
0.5 and 1 were performed, represented by the solid, dashed and dotted lines, respectively, in Figure 5.

Analysis of the role of the pore depth (as controlled by membrane thickness) shows that for a shallow pore depth, $L=1$ (pore aspect ratio $R_{\mathrm{a}} / L=1$ ) and for values of $\gamma=1$ and $\gamma=0.5$, no increase of the DPSV peak currents with increasing preconcentration time within the range of $3 \mathrm{~s}$ to $90 \mathrm{~s}$ was observed (Figure 5A, dotted and dashed lines). A close inspection of the curves simulated for a diffusion coefficient ratio $\gamma=1$ reveals a slight decrease in the DPSV peaks for longer preconcentration times. This decrease might be due to a small overlap of diffusion zones caused by the increase of the diffusion length of the probe ion with the higher diffusion coefficient in the organic phase. However, the effect on the peak current $G_{\mathrm{p}}$ is minimal so that the pores behave essentially independently. Furthermore, the simulated peak currents were smaller than the peak currents computed for the deeper micropores shown in Figures $5 \mathrm{~B}$ to $5 \mathrm{D}$. Although the transfer of ions from the aqueous phase into the organic phase increased with increasing $t_{\mathrm{c}}$ for all values of the pore depth, the amount of ions transferred back from the organic phase was smaller for the shallower pores shown in Figure 5A. With decreasing pore depth, the time for an ion to pass through the pore becomes shorter. Upon passing through the micropores, the transferring ions might dissipate into the trans-membrane space on the organic side and are not readily available to be stripped back into the aqueous phase. This process is most obvious for pore aspect ratios of one and $\gamma=1$ and $\gamma=0.5$, where the peak currents do not increase from the smallest $t_{\mathrm{c}}$ of $3 \mathrm{~s}$ up to $90 \mathrm{~s}$. However, for the smaller diffusion coefficient in the organic phase $(\gamma=0.115$, solid line in Figure 5A) a small increase of the stripping peak current is observed for shorter preconcentration times. Smaller values of $\gamma$ imply a reduced diffusion length in the organic phase compared to the aqueous phase, leading to an accumulation of ions within the micropores and therefore to an increase 
in the stripping peak current, since these ions are more readily available for the stripping process. For example, for the micropores of $L=1$, the $\left|G_{\mathrm{p}}\right|$ values for $\gamma=0.5$ have almost doubled compared to $\gamma=1$. However, in both these cases, the amount of ions transferred across the ITIES into the pore is quickly balanced by the amount of ions leaving the pores on the organic side and an increase in preconcentration time has no effect on the stripping peaks. The "positive" effect of the pore depth on the peak height can clearly be seen in Figure 5B to 5D. For deeper pores, the "saturation" onset of the peak currents with increasing $t_{\mathrm{c}}$ is shifted towards longer preconcentration times. For the deepest pores used in the simulations $(L=8$ $(200 \mu \mathrm{m}))$, Figure 5D), the stripping peaks coincide for the three different $\gamma$ values for preconcentration times of up to $18 \mathrm{~s}$, indicating that due to the deep pores the transferring ions are trapped within the pores, despite their different diffusion length in the organic phase.

Simulations for pore depths identical to the membranes used in experiments and for a $\gamma$ value as evaluated for the transfer of $\mathrm{TEA}^{+}$from aqueous to the gelified organic phase, by comparison to experiments, are shown as the solid line in Figure 5C. The combination of the pore depth of $100 \mu \mathrm{m}$ and the 8.7-fold decrease of the diffusion coefficient caused by the higher viscosity of the gelified organic phase ensures a high efficiency of the stripping process. Replacing the gelified 1,6-DCH by 1,2-dichloroethane, an organic solvent commonly used for electrochemistry at the ITIES, would increase the $\gamma$ coefficient to 0.95 (using a diffusion coefficient of $9.3 \times 10^{-6} \mathrm{~cm}^{2} \mathrm{~s}^{-1}$ for $\mathrm{TEA}^{+}$in 1,2-dichloroethane[37]) and the $\left|G_{\mathrm{p}}\right|-t_{\mathrm{c}}$ curve would resemble the dotted line in Figure 5C, which was calculated for $\gamma=1$. Therefore, the gelification of the organic phase has not only the advantage of stabilisation of the ITIES, but also increases the sensitivity of DPSV measurements. Increasing the membrane thickness or pore depth to $200 \mu \mathrm{m}$ would further enhance the stripping signal, especially for systems with higher ratios of the diffusion coefficients (Figure 5D). The use of gelified 1,6-DCH as 
the organic phase provides a noticeable enhancement of the stripping peak current for preconcentration times $t_{\mathrm{c}}>30 \mathrm{~s}$.

The saturation effect on the stripping peak for longer preconcentration times was also observed in experiments by Lee et al. [22] who used linear sweep stripping voltammetry technique based on a polymer composite membrane (nitrophenyloctylether-PVC) to form micro-liquid/gel interfaces for detection of choline ions. They assumed that the saturation was due to analyte ions travelling through the micropore where linear diffusion prevails and reaching a zone where spherical diffusion prevails, i.e. at the other end of the pore. The ions reaching this zone may not be stripped out as efficiently as those in the micropore [22]. This assumption is supported by the simulations presented here. To illustrate this behaviour, plots of equiconcentration lines for two micropore systems are shown in Figure 6. Figure 6A and 6B presents concentration plots for a pore depth of $L=4\left(l=100 \mu \mathrm{m}, r_{\mathrm{a}}=25 \mu \mathrm{m}\right)$ and $\gamma=0.115$, parameters which were realised in our experimental system. For Figure $6 \mathbf{C}$ and $6 \mathbf{D}$, the parameters $L=1\left(l=25 \mu \mathrm{m}, r_{\mathrm{a}}=25 \mu \mathrm{m}\right)$ and $\gamma=1$, implying no gelification of the organic phase, were used. The equiconcentration lines were calculated at the end of a preconcentration time of $t_{\mathrm{c}}=30 \mathrm{~s}$, just before the stripping of the analyte back into the aqueous phase began. Because of the big concentration range, the areas outside the pores (Figures $6 \mathbf{A}$ and $6 \mathbf{C}$ ) and the areas inside the pores (6B and $6 \mathbf{D})$ are shown separately. The concentration profiles on the aqueous side of the membrane $(Z>0)$ are identical for both pores, since transport of the analyte into the micropores does not depend on the pore depth or the diffusion coefficient of the analyte ions in the organic phase. In both cases, the same amount of analyte crossed the micro-interface during the preconcentration period. However, the distribution of the analyte in the organogel phases is different. For the shallower pore depth and with the non-gelified organic phase $(\gamma=1)$, the spherical diffusion field originating from the pore orifice on the organic side (at $Z=-$ 
1) extends further in a radial direction into the trans-membrane organic phase (Figure 6C) as compared to the profile for the present experimental system (Figure 6B). For the shallower hole, the spherical concentration profile on the organic side of the membrane is almost a mirror image of the spherical concentration profile on the aqueous side, but with complementary concentrations (Figure 6C). This is not the case for the deeper pore and the organo-gel phase shown in Figure 6A. The flat and almost flat equiconcentration lines inside the pores (Figures 6B and 6D) indicate linear and linear-dominated diffusion. Ion accumulation inside the pore is drastically enhanced by increasing the pore depth and by reduction of the diffusion coefficient by gelification of the organic phase, as shown by the lines of high concentrations in Figure 6B. The highest concentration of the analyte is found within the pore close to the wall and in proximity to the ITIES and is a result of the high concentration gradient at the edge of the ITIES (edge effect). For $L=4(l=100 \mu \mathrm{m})$ and $\gamma=0.115$, the concentration of the analyte ion in this region is almost 30 -fold that in the aqueous bulk phase. For the subsequent stripping process, the ions inside the pore are readily available. Increasing $t_{\mathrm{c}}$ beyond $30 \mathrm{~s}$ for the configuration shown in Figure $6 \mathbf{A}$ and $\mathbf{B}$ will still lead to a higher concentration of the analyte ions within the pore and consequently to an increase in the DPSV peak, as shown in Figure 5C, solid line. In contrast, an increase of $t_{\mathrm{c}}$ for the shallower pore will not increase the concentration within the pore and the stripping peak will not increase, as shown in Figure 5A, dotted line. The amount of analyte entering the shallower pore from the aqueous side is almost identical to the loss of ions from the pore by egress on the organic side, illustrated by the symmetry of the spherical concentration profiles on both sides of the membrane (Figure 6C). In such a situation, the analyte concentration within the pore is independent of the preconcentration time and, as a result, a saturation of the stripping peak current occurs. 
A series of experimental DPSVs after preconcentration times of 3, 5, 15, 20, 45, 60 and $90 \mathrm{~s}$ is presented in Figure 7. The inset in Figure 7 shows the comparison of the experimental peak currents to the simulated values as a function of the preconcentration time. The experimental DPSV peak currents show the "saturation" effect for higher preconcentration times, caused by the dissipation of ions into the bulk organic phase. As predicted by the simulation, a preconcentration time of $30-40 \mathrm{~s}$ is sufficient for an optimal peak height. Further increases in the preconcentration time $t_{\mathrm{c}}$ yields only a small increase of current signal $\left|G_{\mathrm{p}}\right|$.

\section{Conclusions}

The combination of $\mu$ ITIES based on regular arrays of micropores in solid state membranes and stripping voltammetric techniques provides an effective platform for ion sensing at liquidliquid interfaces. This combination has been investigated by simulations and compared to experimental data. It was found that an enhancement of the stripping signal was achieved by increasing the length of the pores within which the organic phase is located and by slowing down the diffusion speed of the analyte in the organic phase. The latter is achieved by increasing the viscosity of the organic phase by employing a gelled organic phase. These collective effects are then manifested as enhanced stripping currents because the analyte is not lost from the organic phase within the pores to the bulk organic phase outside the pores but instead is retained within the pores close to the interface and thus is available for the backextraction (stripping) process. The use of deeper pores for formation of $\mu$ ITIES, as is possible by use of silicon membranes [14] rather than polymer membranes [22, 38, 39], and the use of gelled organic phases bring advantages to electroanalytical stripping measurements. 


\section{Acknowledgements}

The support of Science Foundation Ireland (grant number 07/IN.1/B967), the Irish Research

Council for Science Engineering and Technology (PhD scholarship to MDS, grant number RS/2005/122) and the European Commission's Framework Programme 6 (Marie Curie Transfer of Knowledge fellowship to JS, Grant number MTKD-CT-2005-029568)) are gratefully acknowledged. The fabrication facilities employed in this work were supported by the Irish High Education Authority's Programme for Research in Third Level Institutions.

\section{References}

[1] D. W. M. Arrigan, Anal. Lett. 41 (2008) 3233.

[2] F. Reymond, D. Fermin, H. J. Lee, H. H. Girault, Electrochim. Acta 45 (2000) 2647.

[3] Z. Samec, Pure Appl. Chem. 76 (2004) 2147.

[4] P. Vanysek, L. B. Ramirez, J. Chil. Chem. Soc. 53 (2008) 1455.

[5] C. J. Collins, D. W. M. Arrigan, Anal. Chem. 81 (2009) 2344.

[6] A. Berduque, R. Zazpe, D. W. M. Arrigan, Anal. Chim. Acta 611 (2008) 156.

[7] M. D. Scanlon, G. Herzog, D. W. M. Arrigan, Anal. Chem. 80 (2008) 5743.

[8] V. Beni, M. Ghita, D. W. M. Arrigan, Biosens. Bioelectron. 20 (2005) 2097.

[9] D. W. M. Arrigan, M. Ghita, V. Beni, Chemical Communications (2004) 732.

[10] J. Heinze, Angewandte Chemie-International Edition in English 30 (1991) 170.

[11] B. Liu, M. V. Mirkin, Electroanalysis 12 (2000) 1433.

[12] G. Taylor, H. H. J. Girault, J. Electroanal. Chem. 208 (1986) 179.

[13] H. J. Lee, P. D. Beattie, B. J. Seddon, M. D. Osborne, H. H. Girault, J. Electroanal. Chem. 440 (1997) 73.

[14] R. Zazpe, C. Hibert, J. O'Brien, Y. H. Lanyon, D. W. M. Arrigan, Lab Chip 7 (2007) 1732.

[15] J. Strutwolf, M. D. Scanlon, D. W. M. Arrigan, Analyst 134 (2009) 148.

[16] M. D. Scanlon, A. Berduque, J. Strutwolf, D. W. M. Arrigan, Electrochim. Acta (in press, doi:10.1016/j.electacta.2008.12.014).

[17] T. Ohkouchi, T. Kakutani, T. Osakai, M. Senda, Anal. Sci. 7 (1991) 371.

[18] H. Katano, M. Senda, Anal. Sci. 14 (1998) 63.

[19] H. Katano, M. Senda, J. Electroanal. Chem. 496 (2001) 103.

[20] M. Senda, H. Katano, Y. Kubota, Collect. Czech. Chem. Commun. 66 (2001) 445.

[21] M. Senda, H. Katano, M. Yamada, J. Electroanal. Chem. 475 (1999) 90.

[22] H. J. Lee, C. Beriet, H. H. Girault, Anal. Sci. 14 (1998) 71.

[23] H. Katano, H. Tatsumi, M. Senda, Talanta 63 (2004) 185.

[24] T. J. Davies, R. G. Compton, J. Electroanal. Chem. 585 (2005) 63.

[25] H. H. J. Girault, D. J. Schiffrin, J. Electroanal. Chem. 195 (1985) 213.

[26] Z. Samec, J. Electroanal. Chem. 99 (1979) 197.

[27] J. Strutwolf, J. A. Manzanares, D. E. Williams, Electrochem. Commun. 1 (1999) 139.

[28] N. Nishi, S. Imakura, T. Kakiuchi, J. Electroanal. Chem. 621 (2008) 297. 
[29] P. J. Rodgers, S. Amemiya, Anal. Chem. 79 (2007) 9276.

[30] T. Kakiuchi, Y. Teranishi, J. Electroanal. Chem. 396 (1995) 401.

[31] Y. Saito, Rev. Polarogr. 15 (1968) 177.

[32] T. A. Davis, ACM Transactions on Mathematical Software 30 (2004) 196.

[33] K. Ludwig, L. Rajendran, B. Speiser, J. Electroanal. Chem. 568 (2004) 203.

[34] J. L. Melville, R. G. Compton, Electroanalysis 13 (2001) 123.

[35] J. Josserand, J. Morandini, H. J. Lee, R. Ferrigno, H. H. Girault, J. Electroanal. Chem. 468 (1999) 42.

[36] Y. H. Shao, M. V. Mirkin, Anal. Chem. 70 (1998) 3155.

[37] T. Wandlowski, V. Marecek, Z. Samec, R. Fuoco, J. Electroanal. Chem. 331 (1992) 765.

[38] B. Kralj, R. A. W. Dryfe, Phys. Chem. Chem. Phys. 3 (2001) 5274.

[39] S. Peulon, V. Guillou, M. L'Her, J. Electroanal. Chem. 514 (2001) 94. 
Figure legends:

Figure 1. Computational domain and boundary conditions for the simulation of the $\mu$ ITIES array. The boundary condition at the liquid-liquid interface (dotted line) is given by Equations (1) and (2).

Figure 2. Generation of the differential pulse waveform $(\mathbf{C})$ by superimposition of a potential step waveform (A) and a potential pulse waveform (B).

Figure 3. Comparison of experimental and simulated cyclic voltammograms for the transfer of TEA ${ }^{+}$across the ITIES formed at a micropore array. Solid lines: experimental (background subtracted) voltammogram. Open circles: simulated voltammogram. Scan rate: $10 \mathrm{mVs}^{-1}$. For the simulated voltammogram, $\gamma=0.115$ and $\Delta_{\mathrm{o}}^{\mathrm{w}} \varphi^{0^{\prime}}=0.58 \mathrm{~V}$. The current was normalised by the limiting value.

Figure 4. (A) Simulated pulse voltammogram (normalised current versus time), including the current response during the preconcentration step of $t_{\mathrm{c}}=30 \mathrm{~s}$. The inset shows a magnified view of the current response to the potential pulses. (B) The DPSV voltammogram calculated from the voltammogram shown in A. The initial and final potentials were 0.85 and $0.3 \mathrm{~V}$.

Figure 5. Simulated DPSV peak currents as a function of the preconcentration times, for different values of $\gamma=D_{\mathrm{o}} / D_{\mathrm{a}}$ and pore depth $L$. The solid, dashed and dotted lines are simulations for $\gamma=0.115,0.5$, and 1, respectively. The pore depths were: $\mathbf{A}: L=1(25 \mu \mathrm{m})$; B: $L=2(50 \mu \mathrm{m}) ; \mathbf{C}: L=4(100 \mu \mathrm{m})$ and $\mathbf{D}: L=8(200 \mu \mathrm{m})$.

Figure 6. Simulated concentration profiles at the end of the preconcentration time $t_{\mathrm{c}}=30 \mathrm{~s}$ The micro-ITIES is at $Z=0,0<R<1$ (see Figure 1). Parameters for $\mathbf{A}$ and $\mathbf{B}: L=4(l=100 \mu \mathrm{m}$, $\left.r_{\mathrm{a}}=25 \mu \mathrm{m}\right)$ and $\gamma=0.115$; and for $\mathbf{C}$ and $\mathbf{D}: L=1\left(l=25 \mu \mathrm{m}, r_{\mathrm{a}}=25 \mu \mathrm{m}\right)$ and $\gamma=1$. A and $\mathbf{C}$ show equiconcentration lines in the area around the pore, while $\mathbf{B}$ and $\mathbf{D}$ show the concentration distribution within the pores.

Figure 7. Experimental background-subtracted DPSV scans following pre-concentration times of 3, 5, 15, 20, 45, 60 and $90 \mathrm{~s}$ (with increasing absolute peak height). The analyte concentration $\left(\mathrm{TEA}^{+}\right.$) was $150 \mu \mathrm{M}$. The inset shows the experimental peak currents together with simulated DPSV peak currents (solid line) as a function of the pre-concentration time, $t_{\mathrm{c}}$. 
Fig 1:

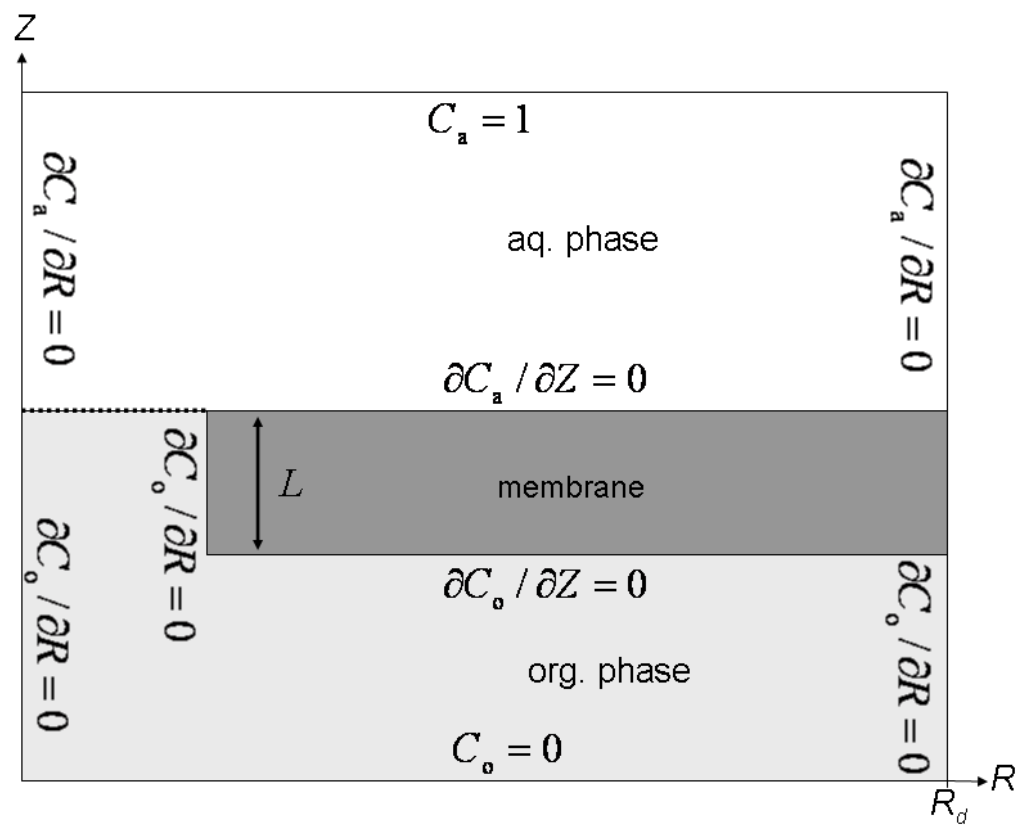

Fig 2:
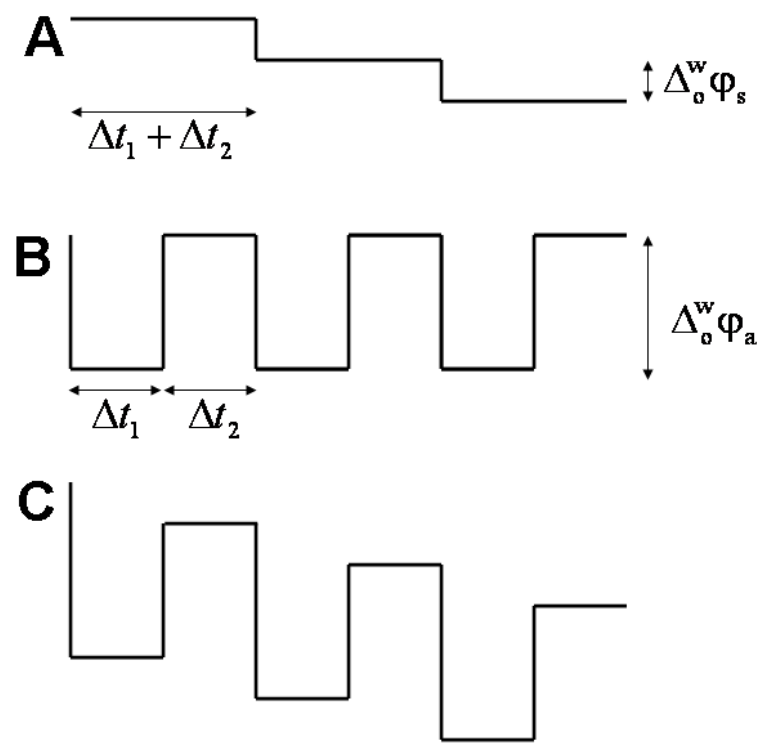
Fig 3:

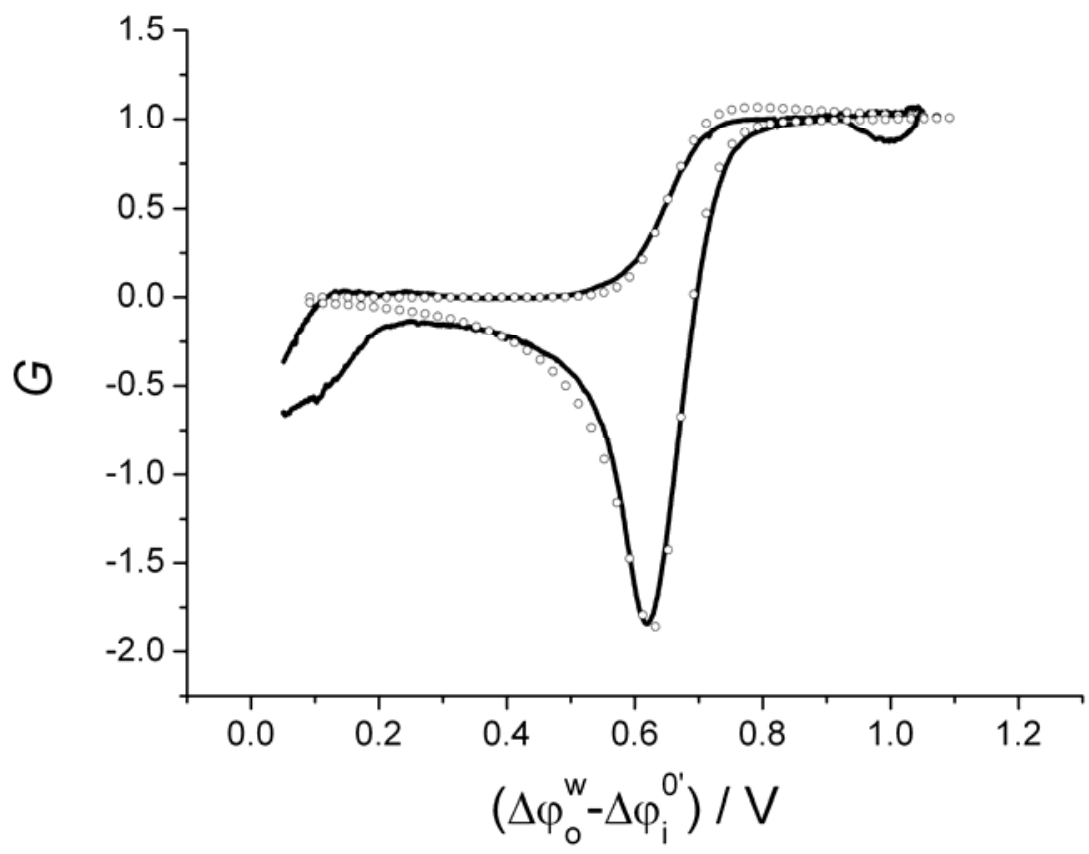

Fig 4A:

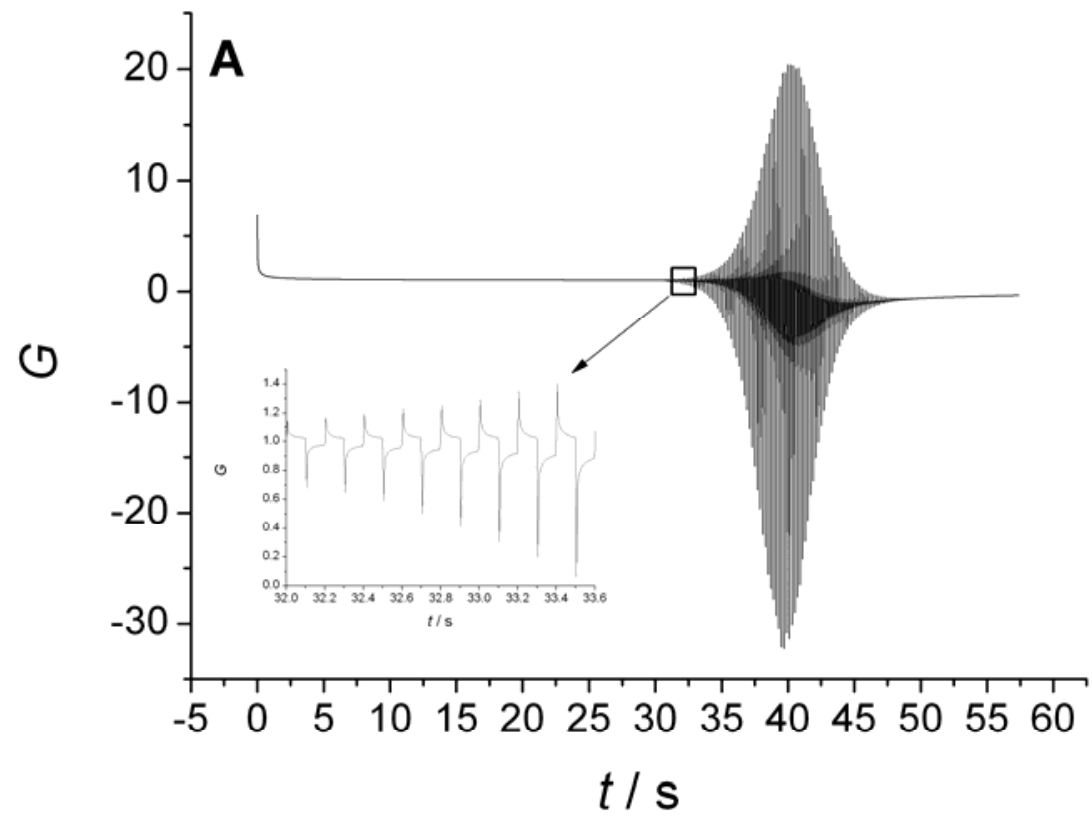


Fig 4B:

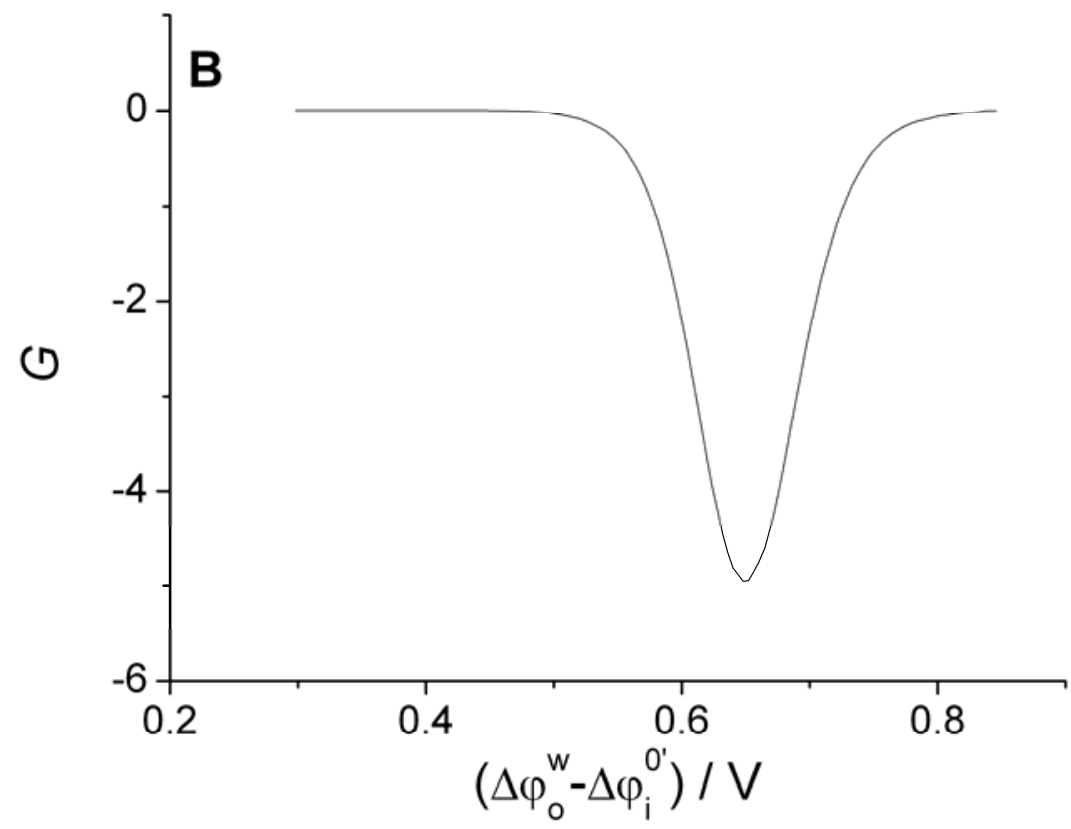

Fig 5:

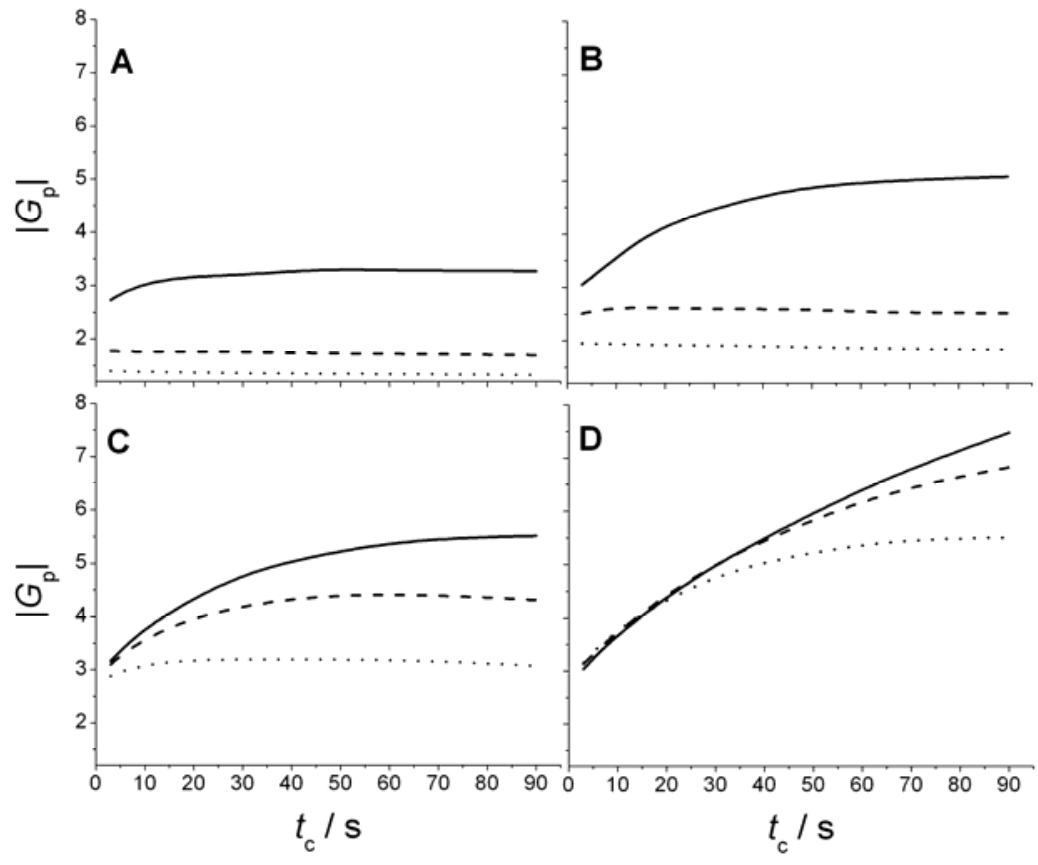


Fig 6A,B:

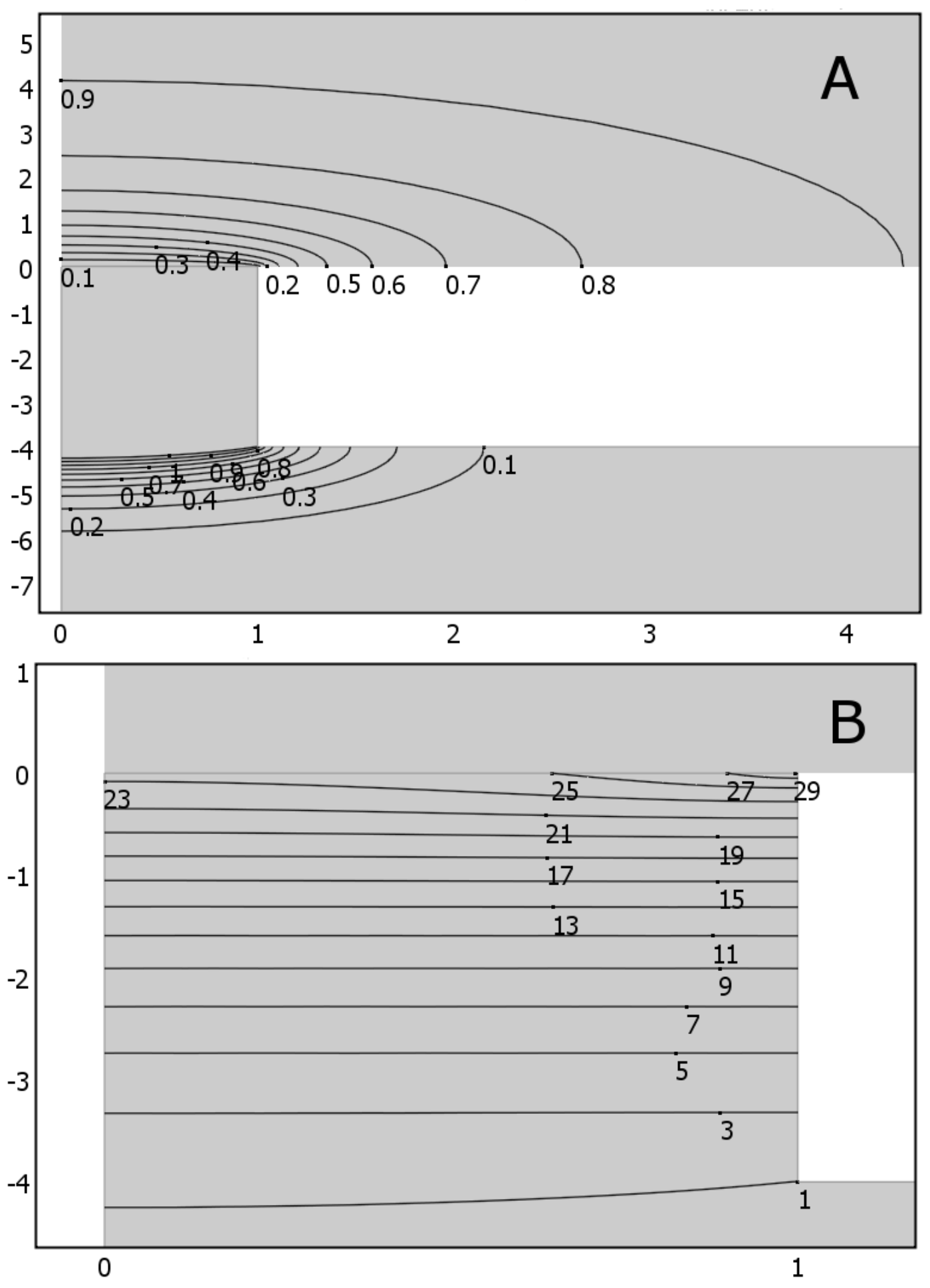


Fig 6C,D:

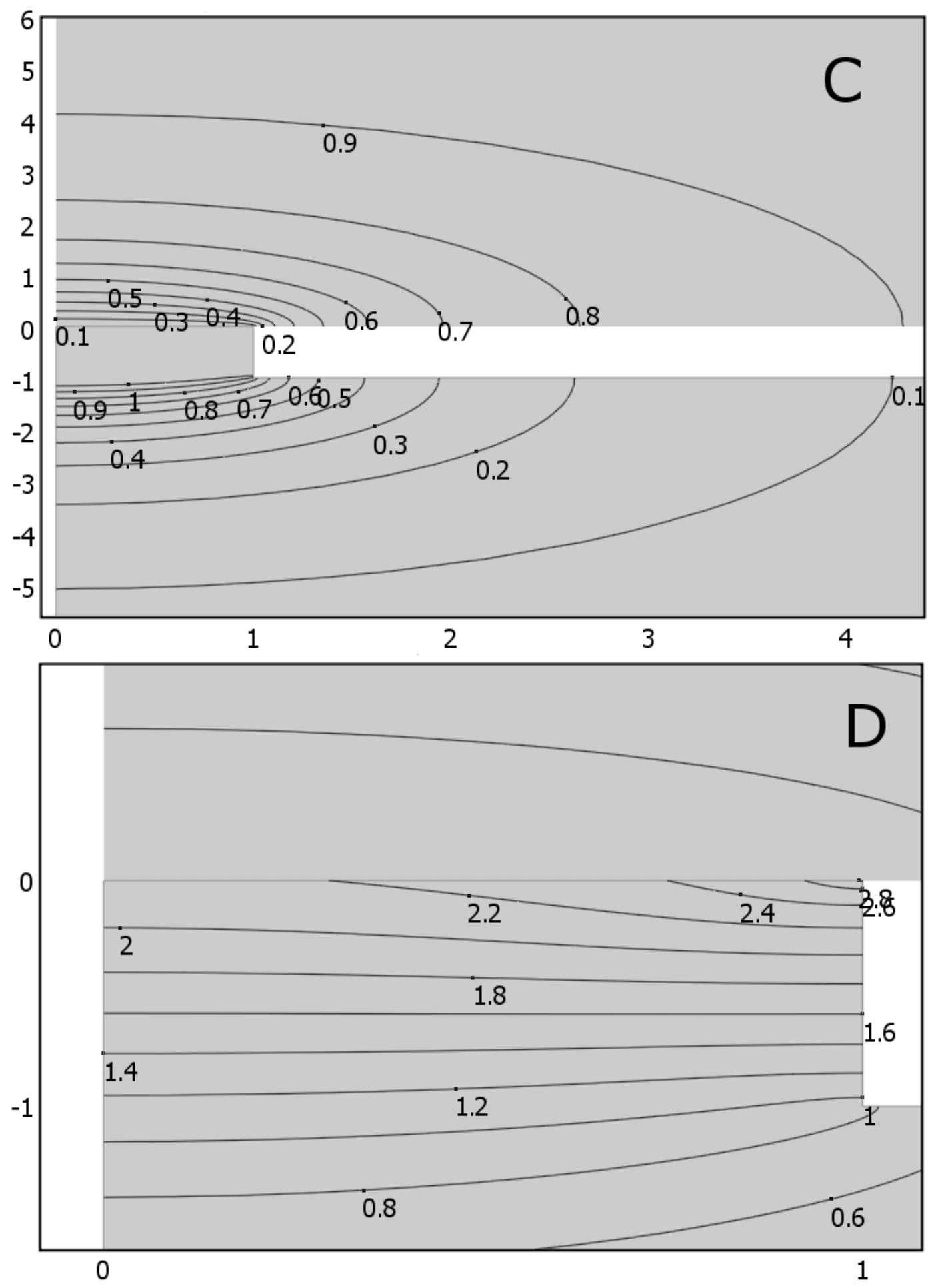


Fig 7:

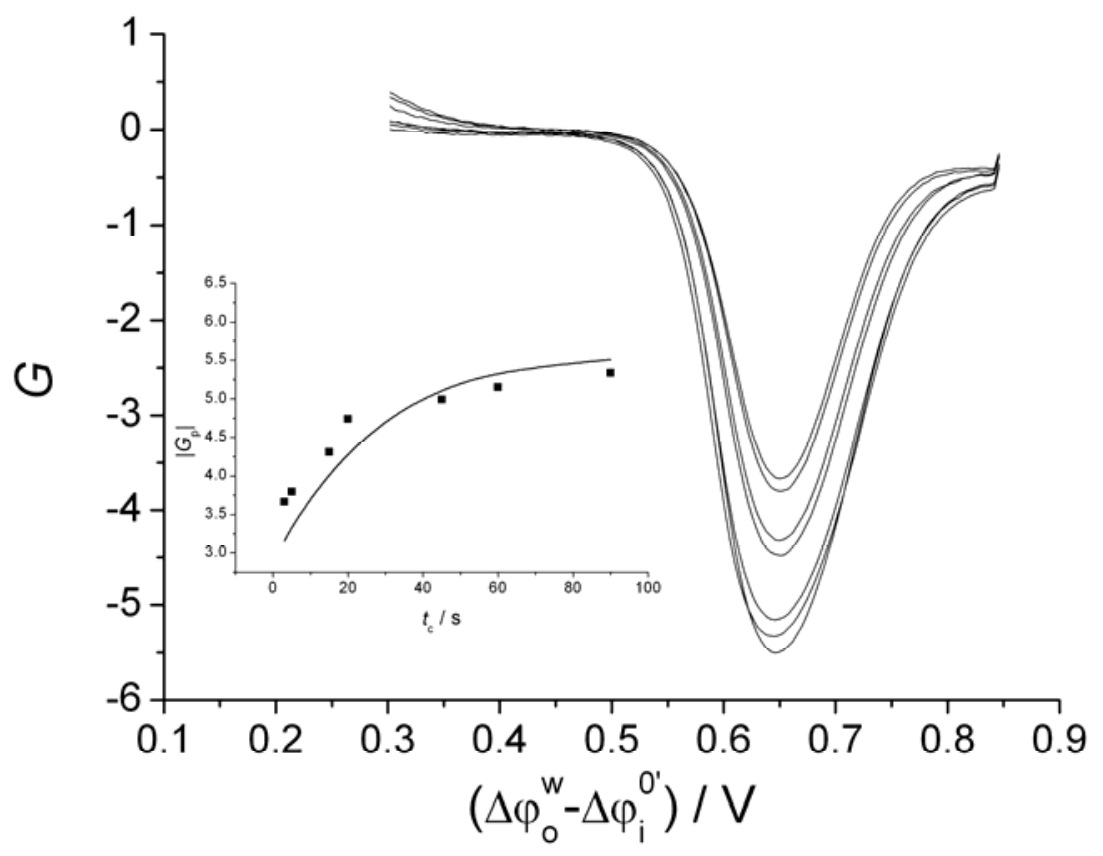

p. 25 . 